\title{
Ethnobotanical resources and traditional skills prevalent among the Tagin community of Arunachal Pradesh, India
}

\author{
Rubu Rinyo', Momang Taram¹, Pallabi K. Hui ${ }^{2}$ and Hui Tag ${ }^{1,3}$ \\ ${ }^{1}$ Plant Systematic \& Ethnobotanical Research Laboratory, Department of Botany, Rajiv Gandhi University, \\ Rono Hills, Doimukh-791112, Arunachal Pradesh, India \\ ${ }^{2}$ Department of Biotechnology, National Institute of Technology (NIT) - Arunachal Pradesh, Yupia-791112, \\ Papum Pare, Arunachal Pradesh, India. \\ ${ }^{3}$ Corresponding Author, e-mail: huitag2008rgu@gmail.com
}

[Received 26.11.2018; Revised 20.12.2018; Accepted 25.12.2018; Published 31.12.2018]

\begin{abstract}
Ethnobotanical field survey was conducted in 10 villages of the Tagin community of Upper Subansiri District of Arunachal Pradesh during the month of May - June 2018 using semistructured questionnaire and focused group discussion methods. The investigation reveals 70 species belonging to 62 genera and 44 plant families along with some unique sets of traditional knowledge and skills associated with diverse use of ethnobotanical resources available in their ethnoecological landscape.
\end{abstract}

Key words: Tagin tribe, Ethnobotany, Traditional Skill, Arunachal Pradesh

\section{INTRODUCTION}

The state of Arunachal Pradesh comprises of 25 districts with a total geographical area of $83,743 \mathrm{sq} \mathrm{km}$ rich in biocultural diversity and traditional knowledge system (Tag \& Das 2007). The Upper Subansiri district is one of the oldest district of Arunachal Pradesh which was created in 1980 with Daporijo as its district headquarters (Figure 1). The district derived its name from the river Subansiri which navigates the whole length of the district and is one of the major tributary of River Brahmaputra in Assam. The district is bounded by Kamle District in South West, West Siang District in the East, Tibet in the North and Kurung Kumey district on the North West (Anonymous 2011). The elevation of the district ranging from $600 \mathrm{~m}$ to $3800 \mathrm{~m}$ from the mean sea level and located between geographical coordinate of $28.5^{\circ}$ and $28.25^{\circ}$ North Latitudes and $93.15^{\circ}$ and $94.20^{\circ}$ East Longitudes. The region is blessed with thick and dense forest comprises of moist alpine, temperate and subtropical forest, and tropical wet evergreen forest in the whole district rich in floral and faunal elements. The district is inhabited by 3 major tribes namely, the Tagin, Nyishi and the Galo, out of which Tagin community has the highest population (Murtem \& Chaudhry 2016). The Tagin community of Upper Subansiri District of Arunachal Pradesh have been using the plants as food, medicinal and cultural materials since time immemorial. Literature studies have revealed that there is lack of published ethnobotanical information available to date on Tagin community. Although the region is rich in plant diversity and traditional knowledge, but there is a lack of published ethnobotanical information on the target community. Therefore, present paper describes ethnobotanical resources and traditional skills prevalent among the Tagin community of Arunachal Pradesh which aims to document the ethnobotanical diversity and associated traditional knowledge system for future sustainable conservation and management practices. 


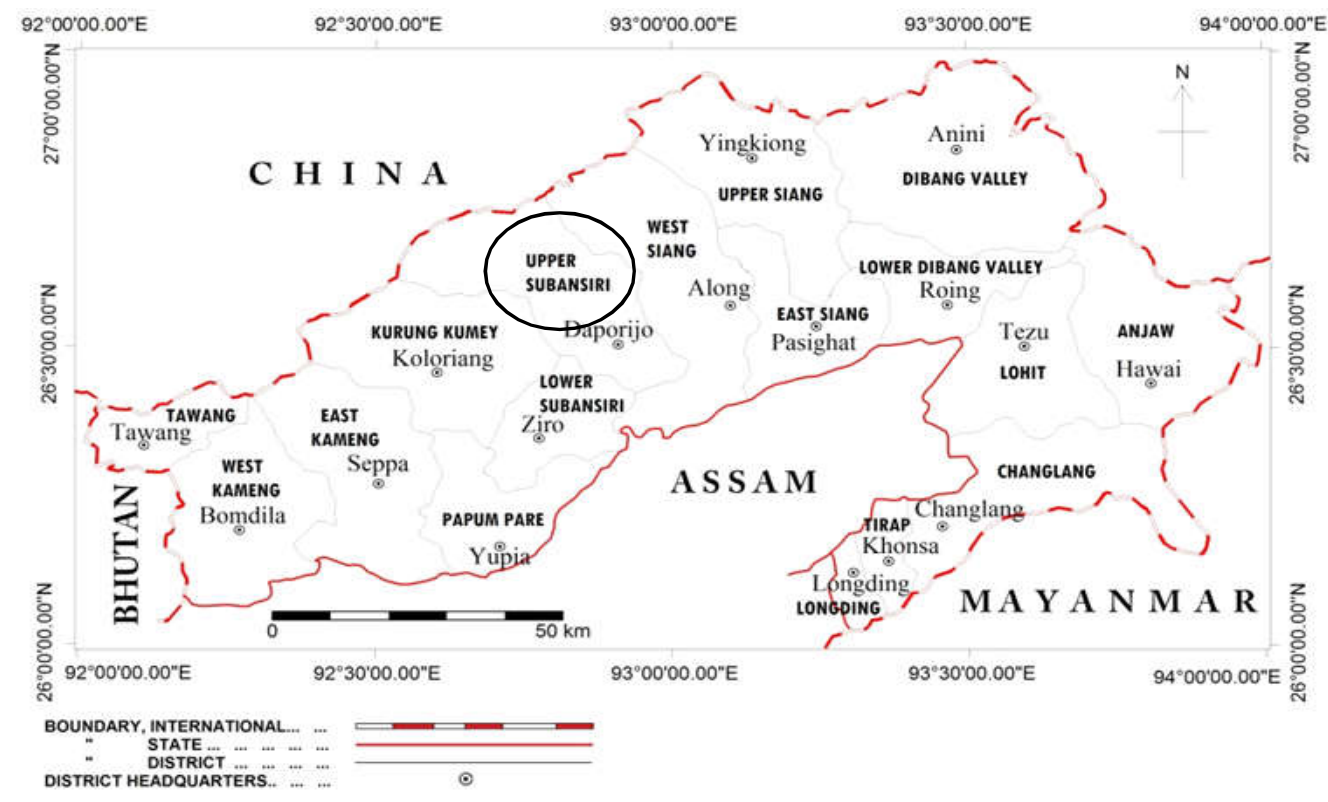

Figure 1. Map showing the location of study area (not to scale)

\section{MATERIALS AND METHODS}

The ethnobotanical survey was carried out in the 10 selected villages of Upper Subansiri District of Arunachal Pradesh, namely, Sippi, Menga, Radding, Chotaropuk, Ronya, Baririjo, Riddi, Pakarijo, Liruk, and Dumporijo during the month of May to June 2018 following the field method suggested by Martin (2008). The traditional knowledge and skills associated with ethnobotanical resources utilization were documented using semi-structured questionnaire and a focused group discussion session conducted in 10 selected villages of rural and semi-urban ethnoecological landscape. In all, 14 informants of age between 25 - 70 were selected for the interview of which 6 were female and 8 males. In addition, Prior Informed Consent (PIC) were obtained from the traditional knowledge holders prior to extensive field study as suggested by Lokho and Narasimhan (2013). During the field visit, translation of local nomenclature of plants and documentation of plant based traditional knowledge were assisted by local knowledge holders of Tagin community who were expertise in their local dialect, indigenous cultural system and local ethnoecological landscape. The ethnobotanical species with local names, parts used, mode of use, habits, and habitats of each plants were recorded in field notebook. The voucher specimens were collected and prepared in the form of herbarium by following Jain \& Rao (1977) method which were deposited in the Herbarium of Arunachal University (HAU), Department of Botany, Rajiv Gandhi University, Rono Hills, Doimukh, Arunachal Pradesh for future reference. Each of the ethnobotanical species were identified through consultation of Standard Floristic Literatures such as Flora of Assam (Kanjilal et al. 1934 - 1940), The Flora of British India (Hooker, 1875 - 1897), e-Flora of China, and eHerbarium of Kew. The currently accepted names were verified in the website www.theplantlist.org hosted by RBG Kew and Missouri Botanical Garden, St. Louis, USA.

\section{RESULT AND DISCUSSION}

The current data on ethnobotanical investigation reveals 70 plant species belonging 62 genera and 44 families (Table 1; Plate - I) which is used by the Tagin community of Upper Subansiri 
District of Arunachal Pradesh. The most dominant plant family recorded is the Asteraceae (7) followed by Arecaceae (6) Solanaceae (4), Lauraceae (3), Polygonaceae (3) and Moraceae (3) (Figure 2). Out of total 70 species of ethnobotanically useful plants recorded, 23 are the herb species, 22 are the shrubs, 19 of trees and 6 are of climbers (Figure 3). The plants with various ethnobotanical uses has been recorded from the local residents which includes edible, medicinal, construction, ritual, livestock medication and poisoning agents.

Table 1. Checklist of ethnobotanical significant plants used by the Tagin community of Upper Subansiri District of Arunachal Pradesh.

\begin{tabular}{|c|c|c|c|c|c|}
\hline $\begin{array}{l}\text { Botanical Name [Family]; } \\
\text { Voucher specimen }\end{array}$ & $\begin{array}{l}\text { Local } \\
\text { Name }\end{array}$ & Habit & $\begin{array}{l}\text { Part } \\
\text { Used }\end{array}$ & $\begin{array}{l}\text { Purpose of } \\
\text { Use }\end{array}$ & Ethnic Uses \\
\hline $\begin{array}{l}\text { Acmella oleracea (L.) R.K. } \\
\text { Jansen [Asteraceae]; Coll. No. } \\
\text { HAU/RR-MT820/ 5.06.2018/ } \\
\text { Sippi }\end{array}$ & Maarsha & Herb & $\begin{array}{l}\text { Flower } \\
\text { and } \\
\text { leaves }\end{array}$ & Edible & $\begin{array}{l}\text { Flower and leaves consumed as } \\
\text { homegrown vegetable. }\end{array}$ \\
\hline $\begin{array}{l}\text { Acmella paniculata (Wall. ex } \\
\text { DC.) R.K. Jansen [Asteraceae]; } \\
\text { Coll. No. HAU/RR-MT821/ } \\
5.06 .2018 / \text { Sippi }\end{array}$ & Byadik & Herb & $\begin{array}{c}\text { Entire } \\
\text { plant }\end{array}$ & Edible & $\begin{array}{l}\text { Entire plant is consumed as } \\
\text { vegetable. }\end{array}$ \\
\hline $\begin{array}{l}\text { Ageratum conizoides (L.) L. } \\
\text { [Asteraceae]; Coll. No. HAU/ } \\
\text { RR-MT823/5.06.2018/Sippi }\end{array}$ & Myora & Herb & Leaves & Medicinal & $\begin{array}{l}\text { Leaves are crushed and the paste is } \\
\text { applied on wound for blood clotting } \\
\text { and wound healing }\end{array}$ \\
\hline $\begin{array}{l}\text { Allium chinense G. Don } \\
\text { [Amaryllidaceae]; Cosll. No. } \\
\text { HAU/RR-MT825/5.06.2018/ } \\
\text { Menga }\end{array}$ & Talap & Herb & $\begin{array}{l}\text { Leaves } \\
\text { and bulb }\end{array}$ & Edible & $\begin{array}{l}\text { Leaves and bulb are edible; It can } \\
\text { be eaten raw as well as cooked }\end{array}$ \\
\hline $\begin{array}{l}\text { Altingia excelsa } \text { Noronha } \\
\text { [Altingeaceae]; Coll. No. HAU/ } \\
\text { RR-MT824/8.06.2018/ } \\
\text { Chotaropuk }\end{array}$ & Siingri & Tree & Trunk & $\begin{array}{l}\text { Bio utensil, } \\
\text { furniture; } \\
\text { construction }\end{array}$ & $\begin{array}{l}\text { Trunk is used for making house } \\
\text { pillar, local furniture and } \\
\text { traditional utensils (mortar) }\end{array}$ \\
\hline $\begin{array}{l}\text { Amomum pterocarpum } \\
\text { Thwaites [Zingiberaceae]; Coll. } \\
\text { No. HAU/RR-T826/8.06.2018/ } \\
\text { Chotaropuk }\end{array}$ & Taleng & Shrub & Leaves & Stuffing & $\begin{array}{l}\text { Leaves are used as packing } \\
\text { material such as for packing rice } \\
\text { and vegetables. }\end{array}$ \\
\hline $\begin{array}{l}\text { Angiopteris evecta (G. Forst.) } \\
\text { Hoffm. [Marattiaceae]; Coll. } \\
\text { No. HAU/RR-MT829/ } \\
\text { 10.07.2018/ Baririjo }\end{array}$ & Tabii & Shrub & Roots & Famine food & $\begin{array}{l}\text { Roots are crushed and paste made } \\
\text { after crushing the roots are shed } \\
\text { dried for 3-4 days. After that, the } \\
\text { paste are packed on leaves and } \\
\text { cooked. The cooked pastes are then } \\
\text { consumed }\end{array}$ \\
\hline $\begin{array}{l}\text { Asystasiella neesiana (Wall.) } \\
\text { Lindau. [Acanthaceae]; Coll. } \\
\text { No. HAU/RR-MT828/ } \\
\text { 5.06.2018/Menga }\end{array}$ & Barse & Herb & leaves & Edible & $\begin{array}{l}\text { Leaves are edible and eaten as a } \\
\text { comestible food }\end{array}$ \\
\hline $\begin{array}{l}\text { Begonia aborensis Dunn } \\
\text { [Begoniaceae]; Coll. No. } \\
\text { HAU/RR-MT827/8.06.2018/ } \\
\text { Chotaropuk }\end{array}$ & Rebe & Herb & stem & $\begin{array}{l}\text { Medicinal; } \\
\text { edible }\end{array}$ & $\begin{array}{l}\text { Stem is eaten as raw and believed } \\
\text { to cure stomach pain }\end{array}$ \\
\hline $\begin{array}{l}\text { Calamus erectus Roxb. } \\
\text { [Arecaceae]; Coll. No. } \\
\text { HAU/RR-MT834/7.06.2018/ } \\
\text { Don }\end{array}$ & Tare & Tree & Stem & Binding & $\begin{array}{l}\text { Stem are locally used as rope for } \\
\text { binding purpose such as for } \\
\text { making boundary, poultry houses, } \\
\text { house construction etc. }\end{array}$ \\
\hline $\begin{array}{l}\text { Calamus flagellum Griff. ex } \\
\text { Mart. [Arecaceae]; Coll. No. } \\
\text { HAU/RR-MT833/7.06.2018/ } \\
\text { Don }\end{array}$ & Iiso & Climber & Stem & Binding & $\begin{array}{l}\text { Stem is used for making local rope } \\
\text { for binding purpose }\end{array}$ \\
\hline $\begin{array}{l}\text { Canarium strictum Roxb. } \\
\text { [Burseraceae]; Coll. No. } \\
\text { HAU/RR-MT831/5.06.2018/ } \\
\text { Menga }\end{array}$ & Silum & Tree & Fruit & Edible & The fruit is edible \\
\hline
\end{tabular}


268 Ethnobotanical resources of Tagin community in Arunachal Pradesh
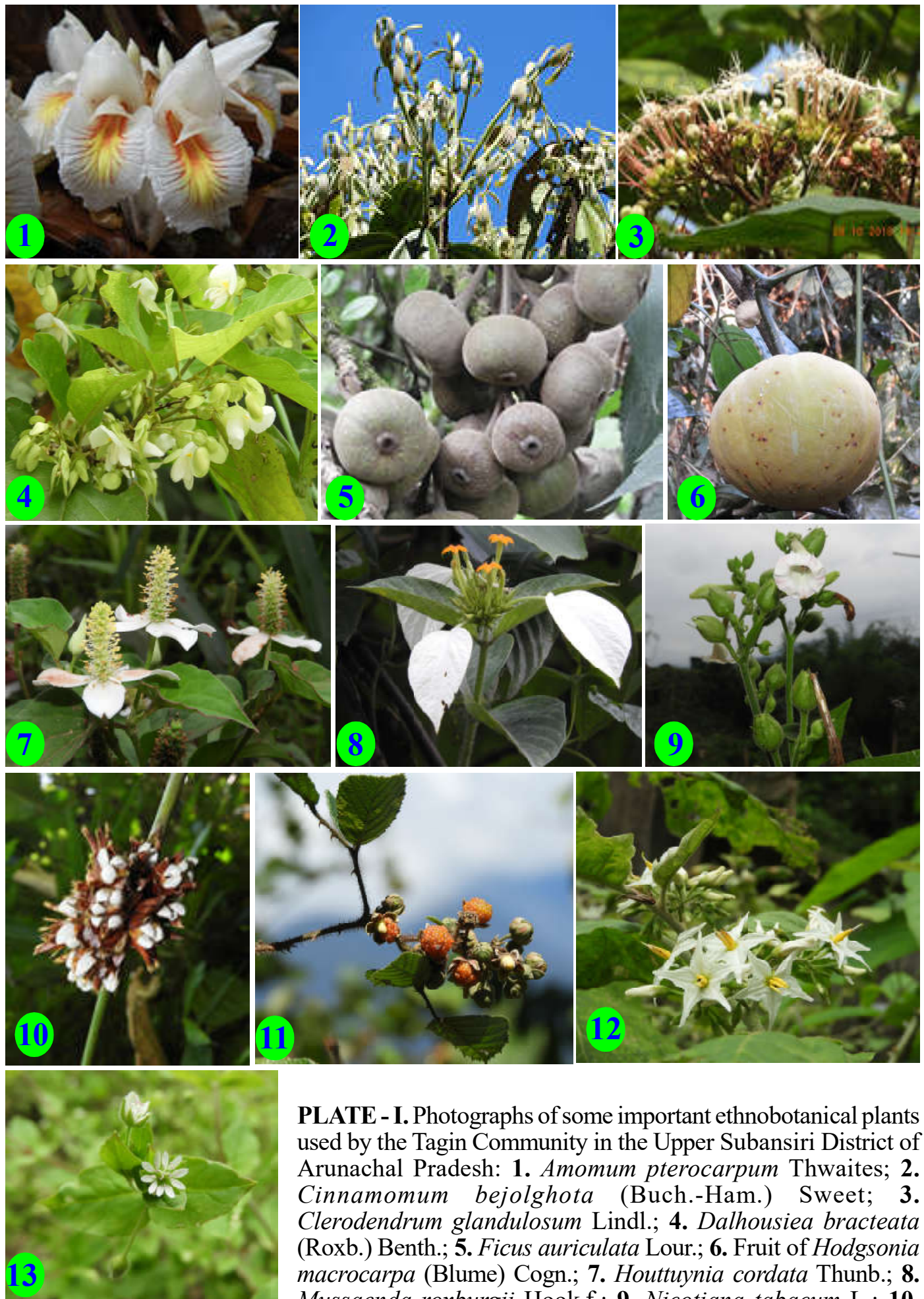

PLATE - I. Photographs of some important ethnobotanical plants used by the Tagin Community in the Upper Subansiri District of Arunachal Pradesh: 1. Amomum pterocarpum Thwaites; 2. Cinnamomum bejolghota (Buch.-Ham.) Sweet; 3. Clerodendrum glandulosum Lindl.; 4. Dalhousiea bracteata (Roxb.) Benth.; 5. Ficus auriculata Lour.; 6. Fruit of Hodgsonia macrocarpa (Blume) Cogn.; 7. Houttuynia cordata Thunb.; 8. Mussaenda roxburgii Hook.f.; 9. Nicotiana tabacum L.; 10. Phrynium pubinerve Blume; 11. Rubus ellipticus Sm.; 12. Solanum torvum Sw.; 13. Stellaria media (L.) Vill. 
Rubu Rinyo et al. 269

\begin{tabular}{|c|c|c|c|c|c|}
\hline $\begin{array}{l}\text { Botanical Name [Family]; } \\
\text { Voucher specimen }\end{array}$ & $\begin{array}{l}\text { Local } \\
\text { Name }\end{array}$ & Habit & $\begin{array}{l}\text { Part } \\
\text { Used }\end{array}$ & $\begin{array}{l}\text { Purpose of } \\
\text { Use }\end{array}$ & Ethnic Uses \\
\hline $\begin{array}{l}\text { Caryota urens L. [Arecaceae]; } \\
\text { Coll. No. HAU/RR-MT832/ } \\
\text { 05.06.2018/Sippi }\end{array}$ & Tamak & Tree & Trunk & Construction & $\begin{array}{l}\text { Trunk is locally used for making } \\
\text { floor of local houses in the tagin } \\
\text { areas; It is also used for making } \\
\text { pathway of local hanging bridges }\end{array}$ \\
\hline $\begin{array}{l}\text { Choerospondias axillaris } \\
\text { (Roxb.) B.L.Burtt \& A.W.Hill } \\
\text { [Anacardiaceae]; Coll. No. } \\
\text { HAU/RR-MT830/5.06.2018/ } \\
\text { Menga }\end{array}$ & Beelam & Tree & Fruit & Edible & $\begin{array}{l}\text { Fruits are eaten as raw. It is sour in } \\
\text { taste }\end{array}$ \\
\hline $\begin{array}{l}\text { Chromolaena odorata }(\text { L.) } \\
\text { R.M.King \& H.Rob. } \\
\text { [Asteraceae]; Coll.No. } \\
\text { HAU/RR-MT881/8.06.2018/ } \\
\text { Chotaropuk }\end{array}$ & $\begin{array}{l}\text { Maling- } \\
\text { Jampak }\end{array}$ & Shrub & Leaves & Medicinal & $\begin{array}{l}\text { Paste of leaves when applied to } \\
\text { cuts and wound, heals and stop } \\
\text { bleeding rapidly; leaves act as a } \\
\text { blood clotting }\end{array}$ \\
\hline $\begin{array}{l}\text { Cinnamomum bejolghota } \\
\text { (Buch.-Ham.) Sweet } \\
\text { [Lauraceae]; Coll. No. } \\
\text { HAU/RR-MT882/12.06.2018/ } \\
\text { Riddi }\end{array}$ & Magob & Tree & Leaves & $\begin{array}{l}\text { Local } \\
\text { beverage }\end{array}$ & $\begin{array}{l}\text { Leaves are used for preparation of } \\
\text { special kind of local beverage } \\
\text { which serves as a local tea among } \\
\text { tagin tribe }\end{array}$ \\
\hline $\begin{array}{l}\text { Clerodendrum glandulosum } \\
\text { Lindl. [Lamiaceae] Coll. No. } \\
\text { HAU/RR-MT883/12.06.2018/ } \\
\text { Riddi }\end{array}$ & $\begin{array}{c}\text { Taapetaa } \\
\text { la / } \\
\text { Taapin }\end{array}$ & Shrub & $\begin{array}{c}\text { Entire } \\
\text { plant }\end{array}$ & Medicinal & $\begin{array}{l}\text { Entire plant is edible and the leaf is } \\
\text { used during lactation period for } \\
\text { curing breast pain and back pain } \\
\text { after heating the leaf on fire; leaves } \\
\text { when consumed are believed to } \\
\text { maintain the blood pressure also }\end{array}$ \\
\hline $\begin{array}{l}\text { Crassocephalum crepidioides } \\
\text { (Benth.) S.Moore [Asteraceae]; } \\
\text { Coll. No. HAU/RR-MT884/ } \\
\text { 12.06.2018/Riddi }\end{array}$ & $\begin{array}{c}\text { Iing- } \\
\text { Kayeng }\end{array}$ & Shrub & Leaves & Edible & $\begin{array}{l}\text { Leaves are consumed as local } \\
\text { vegetable }\end{array}$ \\
\hline $\begin{array}{l}\text { Cyclosorus extensus (Blume) } \\
\text { H. Itô [Thelypteridaceae]; } \\
\text { Coll. No. HAU/RR-MT886/ } \\
\text { 12.06.2018/Riddi }\end{array}$ & Rukdik & Shrub & Leaves & $\begin{array}{l}\text { Livestock } \\
\text { medication }\end{array}$ & $\begin{array}{l}\text { It is believed that placing the } \\
\text { leaves on local poultry houses are } \\
\text { believed to cure viral and bacterial } \\
\text { diseases within }\end{array}$ \\
\hline $\begin{array}{l}\text { Dalhousiea bracteata } \text { (Roxb.) } \\
\text { Benth. [Leguminosae]; Coll. } \\
\text { No. HAU/RR-MT885/ } \\
\text { 06.06.2018/ Radding }\end{array}$ & Tanyom & Shrub & leaves & Ritual & $\begin{array}{l}\text { The tagin people use the leaves of } \\
\text { this plant for ritualistic purpose. }\end{array}$ \\
\hline $\begin{array}{l}\text { Dicranopteris linearis (Burm. } \\
\text { f.) Underw. [Gleicheniaceae]; } \\
\text { Coll. No. HAU/RR-MT888/ } \\
\text { 06.06.2018/Radding }\end{array}$ & Taho & Shrub & Stem & Binding & $\begin{array}{l}\text { Stem is used as a local belt; The } \\
\text { outer part of stem is pulled off and } \\
\text { the inner part of the stem, which is } \\
\text { quite tough, is locally used as a } \\
\text { belt during early days. }\end{array}$ \\
\hline $\begin{array}{l}\text { Dioscorea pentaphylla } \mathrm{L} . \\
\text { [Dioscoriaceae]; Coll. No. } \\
\text { HAU/RR-MT865/11.06.2018/ } \\
\text { Dumporijo }\end{array}$ & Hiili & Climber & tuber & Edible & $\begin{array}{l}\text { Hanging tuber is edible; The tuber } \\
\text { is consumed after boiling }\end{array}$ \\
\hline $\begin{array}{l}\text { Diplazium esculentum (Retz.) } \\
\text { Sw. [Athyriaceae]; Coll. No. } \\
\text { HAU/RR-MT866/12.06.2018/ } \\
\text { Riddi }\end{array}$ & $\begin{array}{l}\text { Pakya- } \\
\text { Raya }\end{array}$ & Shrub & Frond & Medicinal & $\begin{array}{l}\text { Crushed and paste made after } \\
\text { boiling the frond are used for } \\
\text { curing digestive problems }\end{array}$ \\
\hline $\begin{array}{l}\text { Drymaria cordata (L.) Willd. } \\
\text { ex Schult. [Caryophyllaceae]; } \\
\text { Coll. No. HAU/RR-MT880/ } \\
\text { 12.06.2018/Riddi }\end{array}$ & $\begin{array}{l}\text { Bodo- } \\
\text { Bolo }\end{array}$ & Herb & leaves & $\begin{array}{l}\text { Livestock } \\
\text { medication }\end{array}$ & $\begin{array}{l}\text { Crushed /paste of leaves are } \\
\text { applied on the bone of domestic } \\
\text { animals (dog and hen) to heal the } \\
\text { broken bone }\end{array}$ \\
\hline $\begin{array}{l}\text { Duabanga grandiflora (DC.) } \\
\text { Walp. [Lythraceae]; Coll. No. } \\
\text { HAU/RR-MT867/10.06.2018/ } \\
\text { Baririjo }\end{array}$ & Kolok & Tree & Trunk & Construction & $\begin{array}{l}\text { Trunk is particularly used for } \\
\text { building the local houses; Stand or } \\
\text { pillars are usually made }\end{array}$ \\
\hline $\begin{array}{l}\text { Erigeron canadensis L. } \\
\text { [Asteraceae]; Coll. No. } \\
\text { HAU/RR-MT868/8.06.2018/ } \\
\text { Chotaropuk }\end{array}$ & $\begin{array}{l}\text { Bidiing- } \\
\text { Kotar }\end{array}$ & Herb & $\begin{array}{l}\text { Young } \\
\text { shoot }\end{array}$ & Edible & $\begin{array}{l}\text { Young shoot are consumed as a } \\
\text { vegetables }\end{array}$ \\
\hline
\end{tabular}


270 Ethnobotanical resources of Tagin community in Arunachal Pradesh

\begin{tabular}{|c|c|c|c|c|c|}
\hline $\begin{array}{l}\text { Botanical Name [Family]; } \\
\text { Voucher specimen }\end{array}$ & $\begin{array}{l}\text { Local } \\
\text { Name }\end{array}$ & Habit & $\begin{array}{l}\text { Part } \\
\text { Used }\end{array}$ & $\begin{array}{l}\text { Purpose of } \\
\text { Use }\end{array}$ & Ethnic Uses \\
\hline $\begin{array}{l}\text { Fagophyrum esculentum } \\
\text { Moech [Polygoniaceae]; Coll. } \\
\text { No. HAU/RR-MT877/ } \\
\text { 8.06.2018/ Chotaropuk }\end{array}$ & $\begin{array}{l}\text { Hoku- } \\
\text { Paya }\end{array}$ & Herb & leaves & Edible & $\begin{array}{l}\text { Leaves are edible and consumed } \\
\text { as vegetable }\end{array}$ \\
\hline $\begin{array}{l}\text { Ficus auriculata Lour. } \\
\text { [Moraceae]; Coll. No. AU/RR- } \\
\text { MT879/10.06.2018/Baririjo }\end{array}$ & Tau & Tree & Fruits & Edible & Fruits are edible \\
\hline $\begin{array}{l}\text { Ficus hirta Vahl [Moraceae } \\
\text { Coll. No. HAU/RR-MT878/ } \\
\text { 10.06.2018/Baririjo }\end{array}$ & $\begin{array}{l}\text { Kokshi- } \\
\text { Kyarik }\end{array}$ & Shrub & Fruits & Edible & Fruits are edible \\
\hline $\begin{array}{l}\text { Ficus semicordata } \text { Buch.-Ham. } \\
\text { ex Sm. [Moraceae]; Coll. No. } \\
\text { HAU/RR-MT862/10.06.2018/ } \\
\text { Baririjo }\end{array}$ & Takop & Tree & Fruits & Edible & Fruits are edible \\
\hline $\begin{array}{l}\text { Gnetum montanum Markgr. } \\
\text { [Gnetaceae]; Coll. No. } \\
\text { HAU/RR-MT863/7.06.2018/ } \\
\text { Don }\end{array}$ & Tagiing & $\begin{array}{l}\text { Climber } \\
\text { / Liana }\end{array}$ & Bark & Binding & $\begin{array}{l}\text { Fibre of bark is used as ropes for } \\
\text { tying up the local arrow after fire } \\
\text { dried }\end{array}$ \\
\hline $\begin{array}{l}\text { Gynocardia odorata } \text { R.Br. } \\
\text { [Achariaceae]; Coll. No. } \\
\text { HAU/RR-MT864/6.06.2018/ } \\
\text { Radding }\end{array}$ & Siingrit & Tree & Fruit & $\begin{array}{l}\text { Fish } \\
\text { poisoning }\end{array}$ & $\begin{array}{l}\text { Dried fruit are grinded into } \\
\text { powder and used as poisoning } \\
\text { material during fish hunting }\end{array}$ \\
\hline $\begin{array}{l}\text { Gynura cusimbua (D.Don) } \\
\text { S.Moore [Asteraceae]; Coll. } \\
\text { No. HAU/RR-MT861/ } \\
\text { 09.06.2018/Pakarijo }\end{array}$ & Yogin & Herb & $\begin{array}{l}\text { Leaves } \\
\text { and } \\
\text { tender } \\
\text { shoot }\end{array}$ & Edible & $\begin{array}{l}\text { Leaves and tender shoot are edible } \\
\text { and consumed as a vegetable }\end{array}$ \\
\hline $\begin{array}{l}\text { Hodgsonia macrocarpa } \\
\text { (Blume) Cogn. [Cucurbitaceae] } \\
\text { Coll. No. HAU/RR- } \\
\text { MT869/7.06.2018/ Don }\end{array}$ & Tatar & Climber & Seed & Edible & $\begin{array}{l}\text { Cotyledons are eaten after roasted } \\
\text { which is rich in oil. }\end{array}$ \\
\hline $\begin{array}{l}\text { Houttuynia cordata Thunb. } \\
\text { [Saururaceae]; Coll. No. } \\
\text { HAU/RR-MT870/10.06.2018/ } \\
\text { Ronya }\end{array}$ & Hiingya & Herb & $\begin{array}{l}\text { Entire } \\
\text { plant }\end{array}$ & Edible & $\begin{array}{l}\text { Entire plant is edible and } \\
\text { consumed as chutney or salad }\end{array}$ \\
\hline $\begin{array}{l}\text { Hydrocotyle javanica Thunb. } \\
\text { [Araliaceae]; Coll. No. } \\
\text { HAU/RR-MT858/7.06.2018/ } \\
\text { Don }\end{array}$ & $\begin{array}{l}\text { Kojak- } \\
\text { Rotak }\end{array}$ & Herb & Leaves & Edible & $\begin{array}{l}\text { Leaves are boiled and served as } \\
\text { vegetable. }\end{array}$ \\
\hline $\begin{array}{l}\text { Ipomoea batatas (L.) Lam. } \\
\text { [Convolvulaceae]; Coll. No. } \\
\text { HAU/RR-MT859/10.06.2018/ } \\
\text { Ronya }\end{array}$ & $\begin{array}{l}\text { Karya- } \\
\text { Riyamiy } \\
\text { a }\end{array}$ & $\begin{array}{l}\text { Herbace } \\
\text { ous vine }\end{array}$ & Tuber & Edible & $\begin{array}{l}\text { Tuber is sweet in taste and } \\
\text { consumed as food after boiling the } \\
\text { tuber }\end{array}$ \\
\hline $\begin{array}{l}\text { Lagenaria siceraria (Molina) } \\
\text { Standl. [Cucurbitacea]; Coll. } \\
\text { No. HAU/RR-MT860/ } \\
\text { 05.06.2018/Sippi }\end{array}$ & Opum & Climber & Fruit & Bio-utensil & $\begin{array}{l}\text { Dried and hollowed fruit are used } \\
\text { as local jug or container since time } \\
\text { immemorial; It is used as bowls to } \\
\text { drink local wine and also to carry } \\
\text { water }\end{array}$ \\
\hline $\begin{array}{l}\text { Litsea cubeba (Lour.) Pers. } \\
\text { [Lauraceae]; Coll. No. } \\
\text { HAU/RR-MT871/10.06.2018/ } \\
\text { Ronya }\end{array}$ & Tayir & Tree & $\begin{array}{l}\text { Leaves } \\
\text { and fruit }\end{array}$ & Edible & $\begin{array}{l}\text { Leaves have good aroma and it is } \\
\text { consumed raw and also as a salad }\end{array}$ \\
\hline $\begin{array}{l}\text { Livistona jenkinsiana Griff. } \\
\text { [Arecaceae]; Coll. No. } \\
\text { HAU/RR-MT872/10.06.2018/ } \\
\text { Ronya }\end{array}$ & Taak & Tree & Leaves & Construction & $\begin{array}{l}\text { In tagin locality, the leaves are } \\
\text { very frequently used for making } \\
\text { thatch of local houses for human } \\
\text { and also for domestic animals like } \\
\text { pig and hen. }\end{array}$ \\
\hline $\begin{array}{l}\text { Lycopodiella cernua (L.) Pic. } \\
\text { Serm. [Lycopodiaceae]; Coll. } \\
\text { No. HAU/RR-MT873/ } \\
\text { 07.06.2018/Don }\end{array}$ & $\begin{array}{l}\text { Dogu- } \\
\text { Miir }\end{array}$ & $\begin{array}{l}\text { Creepin } \\
\text { g Herb }\end{array}$ & $\begin{array}{l}\text { Entire } \\
\text { plant }\end{array}$ & Ritual & $\begin{array}{l}\text { Plant is used for ritualistic purpose } \\
\text { when a person is sick to cure } \\
\text { different types of diseases. }\end{array}$ \\
\hline $\begin{array}{l}\text { Manihot esculenta Crantz } \\
\text { [Euphorbiaceae]; Coll. No. }\end{array}$ & $\begin{array}{l}\text { Engin- } \\
\text { Sida }\end{array}$ & $\begin{array}{l}\text { Woody } \\
\text { shrub }\end{array}$ & Tuber & Edible & $\begin{array}{l}\text { It is edible and consumed after } \\
\text { cooking as a food. }\end{array}$ \\
\hline
\end{tabular}


Rubu Rinyo et al. 271

\begin{tabular}{|c|c|c|c|c|c|}
\hline $\begin{array}{l}\text { Botanical Name [Family]; } \\
\text { Voucher specimen }\end{array}$ & $\begin{array}{l}\text { Local } \\
\text { Name }\end{array}$ & Habit & $\begin{array}{l}\text { Part } \\
\text { Used }\end{array}$ & $\begin{array}{l}\text { Purpose of } \\
\text { Use }\end{array}$ & Ethnic Uses \\
\hline $\begin{array}{l}\text { Melastoma malabathricum L. } \\
\text { [Melastomataceae]; Coll. No. } \\
\text { HAU/RR-MT875/7.06.2018/ } \\
\text { Liruk }\end{array}$ & Dai-Dasa & Shrub & Fruits & Edible & Fruit is sweet in taste. \\
\hline $\begin{array}{l}\text { Musa balbisiana Colla } \\
\text { [Musaceae ]; Coll. No. } \\
\text { HAU/RR-MT856/6.06.2018/ } \\
\text { Radding }\end{array}$ & kolong & Herb & Leaves & Stuffing & $\begin{array}{l}\text { Green leaves are used as packing } \\
\text { material while making local } \\
\text { beverages; leaves are packed } \\
\text { around all over for effective result }\end{array}$ \\
\hline $\begin{array}{l}\text { Mussaenda roxburgii Hook.f. } \\
\text { [Rubiaceae]; Coll. No. } \\
\text { HAU/RR-MT857/7.06.2018/ } \\
\text { Don }\end{array}$ & $\begin{array}{l}\text { Hak- } \\
\text { Paum }\end{array}$ & Shrub & $\begin{array}{l}\text { Leaves } \\
\text { and } \\
\text { tender } \\
\text { shoot }\end{array}$ & Edible & $\begin{array}{l}\text { Leaves and tender shoot are edible } \\
\text { and consumed as good vegetable } \\
\text { among the villagers. }\end{array}$ \\
\hline $\begin{array}{l}\text { Nicotiana tabacum L. } \\
\text { [Solanaceae]; Coll. No. } \\
\text { HAU/RR-MT889/9.06.2018/ } \\
\text { Pakarijo }\end{array}$ & Dehii & Herb & Leaves & Smoking & $\begin{array}{l}\text { Dried and grinded leaves are used } \\
\text { as local tobacco for smoking and } \\
\text { chewing. }\end{array}$ \\
\hline $\begin{array}{l}\text { Perilla frutescens (L.) Britton } \\
\text { [Lamiaceae]; Coll. No. } \\
\text { HAU/RR-MT876/8.06.2018/ } \\
\text { Chotaropuk }\end{array}$ & $\begin{array}{l}\text { Tanam- } \\
\text { Namji }\end{array}$ & Herb & Seed & Edible & $\begin{array}{l}\text { Seeds are edible and rich in oil } \\
\text { content; crushed seed are cooked } \\
\text { with vegetable to enrich the taste } \\
\text { of the vegetable. }\end{array}$ \\
\hline $\begin{array}{l}\text { Persicaria capitata (Buch.- } \\
\text { Ham. ex D.Don) H.Gross } \\
\text { [Polygoniaceae]; Coll. No. } \\
\text { HAU/RR-MT849/8.06.2018/ } \\
\text { Chotaropuk }\end{array}$ & $\begin{array}{l}\text { Beku- } \\
\text { Yalu }\end{array}$ & Herb & $\begin{array}{l}\text { Whole } \\
\text { plant }\end{array}$ & Fodder & $\begin{array}{l}\text { Entire plant of is given as a fodder } \\
\text { for pig. }\end{array}$ \\
\hline $\begin{array}{l}\text { Persicaria hydropiper (L.) } \\
\text { Delarbre [Polygoniaceae]; Coll. } \\
\text { No. HAU/RR-MT890/ } \\
\text { 09.06.2018/Pakarijo }\end{array}$ & Hosum & Herb & $\begin{array}{l}\text { Whole } \\
\text { plant }\end{array}$ & $\begin{array}{l}\text { Fish } \\
\text { poisoning }\end{array}$ & It is used for fish poisoning. \\
\hline $\begin{array}{l}\text { Phoebe cooperiana U.N } \\
\text { Kanjilal ex A.Das [Lauraceae]; } \\
\text { Coll. No. HAU/RR-MT850/ } \\
\text { 06.06.2018/Radding } \\
\end{array}$ & Sechar & Tree & Fruit & Edible & Fruits are edible \\
\hline $\begin{array}{l}\text { Phrynium pubinerve } \text { Blume } \\
\text { [Marantaceae]; Coll. No. } \\
\text { HAU/RR-MT } 851 / 7.06 .2018 / \\
\text { Don }\end{array}$ & Kokam & Herb & Leaves & Stuffing & $\begin{array}{l}\text { Leaves are widely used for packing } \\
\text { purposes. }\end{array}$ \\
\hline $\begin{array}{l}\text { Pinanga gracilis Blume } \\
\text { [Arecaceae ]; Coll. No. } \\
\text { HAU/RR-MT854/7.06.2018/ } \\
\text { Don }\end{array}$ & Tachar & Tree & $\begin{array}{l}\text { Whole } \\
\text { plant }\end{array}$ & Ritual & $\begin{array}{l}\text { Entire plant is used during } \\
\text { ritualistic purpose. }\end{array}$ \\
\hline $\begin{array}{l}\text { Piper pedicellatum } \text { C. DC. } \\
\text { [Piperaceae]; Coll. No. } \\
\text { HAU/RR-MT852/05.06.2018/ } \\
\text { Sippi }\end{array}$ & Yarii & Shrub & Leaves & Edible & $\begin{array}{l}\text { Boiled leaves are served as } \\
\text { vegetable. }\end{array}$ \\
\hline $\begin{array}{l}\text { Portulaca oleracea } \text { L. } \\
\text { [Portulacaceae]; Coll. No. } \\
\text { HAU/RR-MT853/9.06.2018/ } \\
\text { Pakarijo }\end{array}$ & Tadar-oo & Herb & $\begin{array}{l}\text { Whole } \\
\text { plant }\end{array}$ & Edible & $\begin{array}{l}\text { Plant is sour in taste and the entire } \\
\text { plant is consumed as vegetable. }\end{array}$ \\
\hline $\begin{array}{l}\text { Pouzolzia hirta Blume ex } \\
\text { Hassk. [Urticaceae]; Coll. No. } \\
\text { HAU/RR-MT855/9.06.2018/ } \\
\text { Pakarijo }\end{array}$ & Oik & Herb & Leaves & $\begin{array}{l}\text { Edible; } \\
\text { medicinal }\end{array}$ & $\begin{array}{l}\text { Leaves are consumed as a good } \\
\text { vegetable; It is believed to be rich } \\
\text { in vitamin and increases the } \\
\text { hemoglobin level. }\end{array}$ \\
\hline $\begin{array}{l}\text { Pteris tripartita } \text { Sw. } \\
\text { [Pteridaceae]; Coll. No. } \\
\text { HAU/RR-MT843/10.06.2018/ } \\
\text { Baririjo }\end{array}$ & $\begin{array}{c}\text { Tao- } \\
\text { Kapung }\end{array}$ & Shrub & $\begin{array}{l}\text { Tender } \\
\text { leaves }\end{array}$ & Edible & $\begin{array}{l}\text { Tender shoots are consumed as } \\
\text { vegetable. }\end{array}$ \\
\hline $\begin{array}{l}\text { Ricinus communis L. } \\
\text { [Euphorbiaceae]; Coll. No. } \\
\text { HAU/RR-MT888/6.06.2018/ } \\
\text { Radding }\end{array}$ & $\begin{array}{l}\text { Parok- } \\
\text { Iikana }\end{array}$ & Shrub & Leaves & $\begin{array}{l}\text { Fodder; } \\
\text { livestock } \\
\text { medication }\end{array}$ & $\begin{array}{l}\text { Leaves are used as fodder for } \\
\text { caterpillar; caterpillar is fed on this } \\
\text { plant for generating silk; the bark } \\
\text { of long petiole is used as a bandage } \\
\text { to cure the fracture bond of } \\
\text { chicken. }\end{array}$ \\
\hline
\end{tabular}


272 Ethnobotanical resources of Tagin community in Arunachal Pradesh

\begin{tabular}{|c|c|c|c|c|c|}
\hline $\begin{array}{l}\text { Botanical Name [Family]; } \\
\text { Voucher specimen }\end{array}$ & $\begin{array}{l}\text { Local } \\
\text { Name }\end{array}$ & Habit & $\begin{array}{l}\text { Part } \\
\text { Used }\end{array}$ & $\begin{array}{l}\text { Purpose of } \\
\text { Use }\end{array}$ & Ethnic Uses \\
\hline $\begin{array}{l}\text { Rubus ellipticus } \text { Sm. } \\
\text { [Rosaceae]; Coll. No. } \\
\text { HAU/RR-MT844/06.06.2018/ } \\
\text { Radding }\end{array}$ & Tae & Shrub & Fruit & Edible & $\begin{array}{l}\text { Fruit is sweet in taste and } \\
\text { consumed raw. }\end{array}$ \\
\hline $\begin{array}{l}\text { Rubus niveus Thunb. } \\
\text { [Rosaceae]; Coll. No. } \\
\text { HAU/RR-MT845/06.06.2018/ } \\
\text { Radding }\end{array}$ & Tae & Shrub & Fruit & Edible & Fruit is sweet in taste. \\
\hline $\begin{array}{l}\text { Saurauia punduana } \text { Wall. } \\
\text { [Actinidaceae]; Coll. No. } \\
\text { HAU/RR-MT846/9.06.2018/ } \\
\text { Pakarijo }\end{array}$ & Hinchii & Tree & $\begin{array}{c}\text { Entire } \\
\text { plant }\end{array}$ & Ritual & $\begin{array}{l}\text { Fruits are edible and the entire } \\
\text { plant is used for ritualistic purpose; } \\
\text { The plant is believed to be sacred } \\
\text { for using in different ritualistic } \\
\text { resolution }\end{array}$ \\
\hline $\begin{array}{l}\text { Sauropus androgynus (L.) } \\
\text { Merr. [Phyllanthaceae]; Coll. } \\
\text { No. HAU/RR-MT847/ } \\
\text { 05.06.2018/Sippi }\end{array}$ & Oo-Toko & shrub & Leaves & Edible & Leaves are taken as vegetable. \\
\hline $\begin{array}{l}\text { Solanum torvum Sw. } \\
\text { [Solanaceae]; Coll. No. } \\
\text { HAU/RR-MT848/05.06.2018/ } \\
\text { Sippi }\end{array}$ & $\begin{array}{l}\text { Samatae/ } \\
\text { Byake }\end{array}$ & Shrub & Fruit & $\begin{array}{l}\text { Edible; } \\
\text { medicinal }\end{array}$ & $\begin{array}{l}\text { Crushed fruits are applied on the } \\
\text { teeth for curing tooth ache; Fruit } \\
\text { are also consumed as chutney. }\end{array}$ \\
\hline $\begin{array}{l}\text { Solanum americanum Mill. } \\
\text { [Solanaceae]; Coll. No. } \\
\text { HAU/RR-MT841/9.06.2018/ } \\
\text { Pakarijo }\end{array}$ & Horee & Shrub & Leaves & Edible & $\begin{array}{l}\text { Leaves are boiled and consumed } \\
\text { as a good vegetable }\end{array}$ \\
\hline $\begin{array}{l}\text { Solanum spirale } \text { Roxb. } \\
\text { [Solanaceae]; Coll. No. HAU/ } \\
\text { RR-MT842/11.06.18/ } \\
\text { Dumporijo }\end{array}$ & $\begin{array}{l}\text { Sucha- } \\
\text { Kaya }\end{array}$ & Shrub & Fruit & Medicinal & $\begin{array}{l}\text { Among the tagin tribe, the fruit is } \\
\text { believed to cure diarrhea and high } \\
\text { blood pressure when consumed } \\
\text { after boiled }\end{array}$ \\
\hline $\begin{array}{l}\text { Spondias pinnata (L. f.) Kurz } \\
\text { [Anacardiaceae]; Coll. No. } \\
\text { HAU/RR-MT840/10.06.2018/ } \\
\text { Ronya }\end{array}$ & Kodum & Tree & Stem & Construction & $\begin{array}{l}\text { Stem is used for the construction of } \\
\text { pillar of local houses in the tagin } \\
\text { regions }\end{array}$ \\
\hline $\begin{array}{l}\text { Stellaria media (L.) Vill. } \\
\text { [Caryophyllaceae]; Coll. No. } \\
\text { HAU/RR-MT837/10.06.2018/ } \\
\text { Ronya }\end{array}$ & Meedam & Herb & $\begin{array}{l}\text { Whole } \\
\text { plant }\end{array}$ & Edible & $\begin{array}{l}\text { whole plant is consumed as } \\
\text { vegetable }\end{array}$ \\
\hline $\begin{array}{l}\text { Sterculia hamiltonii (Kuntze) } \\
\text { Adelb. [Malvaceae]; Coll. No. } \\
\text { HAU/RR-MT839/11.06.2018/ } \\
\text { Dumporijo }\end{array}$ & Siinglam & Tree & Seed & Edible & Seeds are edible \\
\hline $\begin{array}{l}\text { Thysanolaena latifolia (Roxb. } \\
\text { ex Hornem.) Honda [Poaceae]; } \\
\text { Coll. No. HAU/RR-MT838/ } \\
\text { 11.06.2018/Dumporijo }\end{array}$ & Taji & Herb & $\begin{array}{l}\text { Entire } \\
\text { plant }\end{array}$ & Local Broom & $\begin{array}{l}\text { Entire inflorescences is used as } \\
\text { broom after matured }\end{array}$ \\
\hline $\begin{array}{l}\text { Wallichia oblongifolia } \text { Griff. } \\
\text { [Arecaceae]; Coll. No. } \\
\text { HAU/RR-MT836/11.06.2018/ } \\
\text { Dumporijo }\end{array}$ & Tase & Shrub & $\begin{array}{l}\text { Black } \\
\text { fibres }\end{array}$ & Handicrafts & $\begin{array}{l}\text { People of tagin tribe uses the black } \\
\text { fibres for making local bags called } \\
\text { 'Raaming'; They carry this bag } \\
\text { during rainy days to protect } \\
\text { themselves from rainfall. }\end{array}$ \\
\hline $\begin{array}{l}\text { Zanthoxylum rhetsa } \text { DC. } \\
\text { [Rutaceae]; Coll. No. } \\
\text { HAU/RR-MT835/06.06.2018/ } \\
\text { Radding }\end{array}$ & Honyir & Tree & Leaves & $\begin{array}{l}\text { Fish } \\
\text { poisoning; } \\
\text { edible }\end{array}$ & $\begin{array}{l}\text { Leaves are crushed into powder } \\
\text { and used for fish poisoning; The } \\
\text { leaves are also consumed by the } \\
\text { local people as a vegetable; Leaves } \\
\text { have a strong aroma and believed } \\
\text { to enhance the taste of food. }\end{array}$ \\
\hline
\end{tabular}

Wild edible plants: Of the total plant recorded, 37 plant species are typically used as wild edible food by the local residents. Some of the common and most frequently used edible plants are Acmella paniculata, Crassocephalum crepidioides, Litsea cubeba, Mussaenda roxburgii, Phoebe cooperiana, Piper pedicellatum, Pouzolzia hirta, Solanum torvum, etc. 


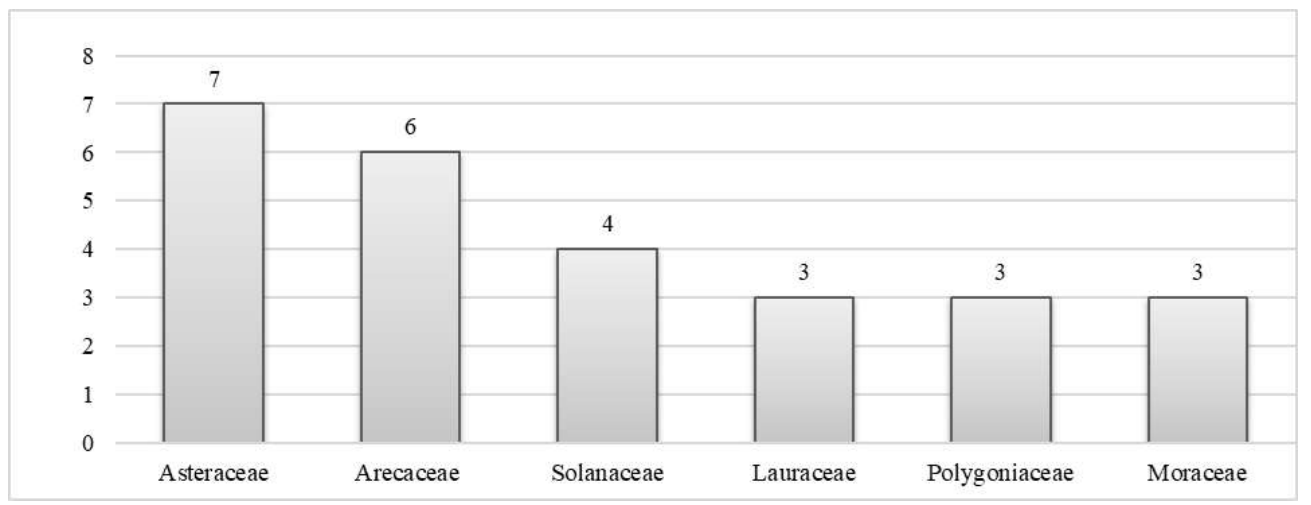

Figure 2. Major plant families of ethnobotanical significance used by the Tagin community of Upper Subansiri District of Arunachal Pradesh

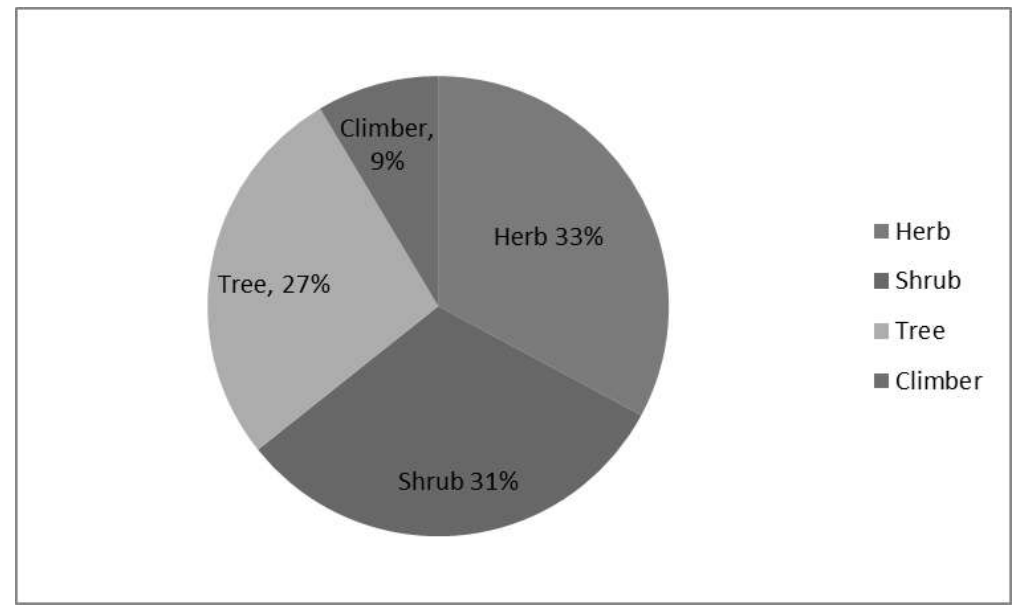

Figure 3. Habit groups of ethnobotanically useful plants used by the Tagin community

Medicinal plants: The study revealed 8 medicinally useful plants which were used by the local residents of rural localities for the treatment of various ailments such as diarrhea, toothache, stomach pain, blood pressure, blood clotting, breast and back pain during lactation period. Some of the significant plant species recorded includes Begonia aborensis, Chromolaena odorata, Clerodendrum glandulosum, Solanum spirale, etc.

Plants used for construction: Tree species like Altingia excelsa, Caryota urens, Duabanga grandiflora, Livistona jenkinsiana and Spondias pinnata were found to be used for the housing and godown construction purposes.

Plants used for local rituals: The Tagin community living in both rural and semi-urban localities use some plant species in traditional rites and rituals ceremony. The total of 4 plants species has been recorded which include Dalhousiea bracteata, Pinanga gracilis, Bambusa stricta and Saurauia armata.

Plants used for Livestock medication: The domestic livestock feds on the pastoral areas of Tagin community which are often infected by worms and other diseases. A total of 3 plants have been shown as livestock medication which include Cyclosorus extensus, Drymaria cordata, and Ricinus communis. 


\section{CONCLUSION}

From present findings, it is concluded that the ethnobotanical resources and associated traditional knowledge and skills prevalent among the Tagin community of Upper Subansiri District is rich and diverse which they have acquired over the generations through oral traditional transmitted from their ancestors. The traditional knowledge and skills prevalent among the community is relevant to their ethnoecological and cultural landscape which help them sustaining their livelihood since millennia. The ethnobotanical resources reported are utility in nature and have economic, ecological and cultural significance. Further scientific studies on food and medicinal plants reported to be used by the target community would yield valuable information about bioactive compounds of economics and commercial significance.

\section{Acknowledgements}

The authors are thankful to NIF and SERB, Department of Science and Technology, Government of India for the financial support through project funding through third and fourth author. The authors are also thankful to all the local informants for having sharing their valuable traditional knowledge during focused group discussions.

\section{LITERATURE CITED}

Anonymous, 2011. Statistical abstract of Arunachal Pradesh. Publication of Directorate of Economics and Statistics, Govt. of Arunachal Pradesh, Itanagar. Pp. 2 - 75.

Gary, J. M. 2008. Ethnobotany: A Methods Manual. Earthscan, 8 - 12 Camden Hight Street, London UK.

Hooker, J.D. 1872. The Flora of British India, Vols.1 - 7, L. Reeve \& Co., London.

Jain, S.K. \& Rao, R.R.1977. A Handbook of Field and Herbarium Methods. Today's \& Tomorrow's Printer and Publishers, New Delhi.

Kanjilal, UN; Kanjilal, PC \& Das, A. 1934 - 1940. Flora of Assam, Vol. 1 - 5. Published by Govt. of Assam.

Lokho, K \& Narsimhan, D. 2013. Ethnobotany of Mao-Naga Tribe of Manipur, India. Pleione $7(2): 314-324$.

Murtem, G. \& Chaudhry, P. 2016. An ethnobotanical study of medicinal plants used by the tribes of Upper Subansiri District of Arunachal Pradesh, India. Amer. J. Ethnomed. 3(3): $35-49$.

Tag, H \& Das, A.K. 2007. Significant plant used by the Nyishi tribe of Arunachal Pradesh. Northeast India. In: A.P. Das \& A.K. Pandey (eds.), Advances in Ethnobotany. Bishen Singh and Mahindra Pal Singh. Dehradun. Pp. 43 - 50.

www.theplantlist.org

www.efloras.org 